\title{
From Policy to Practice - Education in Infection Prevention and Control
}

\author{
Shaheen Mehtar, Frederick Marais and Marina Aucamp \\ Unit for Infection Prevention and Control, Tygerberg Academic Hospital and Division of Community Health, \\ Faculty of Health Sciences, Stellenbosch University, Cape Town, South Africa
}

doi: 10.3396/ijic.V7i2.016.11

\section{Introduction}

Education is pivotal to the outcome of effective healthcare-associated training programmes and nowhere is this more apparent than in infection prevention and control (IPC). ${ }^{1}$ Undergraduate training in healthcare is usually based on the theory of clinical activity and the practical aspects are learnt through apprenticeship. ${ }^{2}$ The curriculum is packed into an intensive four or five years mainly focusing on clinical specialities such as surgery, medicine, paediatrics, obstetrics and gynaecology and chronic diseases. Most of these clinical practices are procedure based while IPC, in its essence, is based on processes which encompass, but are not exclusively attributed to, procedures alone. The basics of IPC principles, such as hand hygiene, require all healthcare practitioners (and others) to consider IPC as an integral part of their lives and working practice.

While IPC plays a major role in outcome of clinical practice, there are few formal IPC courses that cover this essential aspect of patient care in low to middle income (LMI) countries. ${ }^{3}$ IPC training is seen as being part of clinical practice and usually is taught after the students have completed most of their undergraduate training. In some countries, IPC falls under the nursing umbrella and therefore medical students and doctors do not get the same level of training although they, too, work in clinical areas and carry out an equal number of, if not more, clinical procedures. These lines of demarcation, especially in LMI countries, leave a yawning gap in knowledge required to implement good IPC policies. ${ }^{4}$

IPC necessitates adult education and therefore faces challenges of changing practice which has been ingrained over years. This paper addresses methods of teaching which may be helpful in imparting evidencebased knowledge and understanding of the basic principles of IPC at different levels of healthcare practice towards changing behaviour especially when starting IPC education programmes.

\section{Starting an education programme - baseline IPC assessment}

In order for IPC education to have an impact, measured outcomes such as a reduction in healthcare-associated infection (HAl) or reduction in cost of healthcare should be considered. The first prerequisite is a surveillance programme, albeit a simple one. Before starting an education programme, it is worthwhile considering carrying out a baseline IPC assessment of the current level of knowledge, and the extent of HAI. This could be aimed at a speciality, a procedure or a particular pathogen as demonstrated by several studies..$^{5,6,7}$ 
A context-specific questionnaire which covers the current IPC provision for healthcare delivery in the facility should be established. The IPC structure such as existing/available programme(s), number of IPC personnel, their qualifications, presence of an IPC policy and IPC committee (number of times it meets) and attendees (inclusion of all local key stakeholders) should be assessed. It is also important to record the level and extent of IPC training ${ }^{3}$ provided to doctors, nurses and other health care workers. These might be formal or informal, the latter usually happens during ward visits and clinical discussions. If surveillance information regarding $\mathrm{HAl}$ infection rates exists it should be made available and the method of collecting such data noted. Baseline information is essential to guide the development of a relevant education programme, addressing local needs with local solutions.

\section{Setting up an IPC education programme}

Rooted in the baseline information gathered from the health facilities, the training programme can be tailor made to the local healthcare environment. Contextual appropriateness of the training curriculum is critical toward sustainable local capacity building. Training should be facilitated by qualified IPC practitioners. Where such resources do not exist, external tutors with this expertise should be invited to conduct training. In countries with developed IPC programmes, there is no shortage of expertise; however in LMI countries the latter may be necessary initially until local expertise is built up. ${ }^{8}$

Local population and disease profiles greatly influence the IPC programme and should cover the principles of IPC with a particular emphasis on the local conditions. For example, in South Africa, tuberculosis and HIV affect the outcome of IPC programmes and therefore have to be taken into account. In the Near and Far East, dengue is a viral haemorrhagic fever which is widespread. In the Middle East and parts of North Africa, hepatitis $\mathrm{B}$ and $\mathrm{C}$ dominate the population disease profile. Comparatively, in developed countries IPC practitioners focus on the high rates of meticillinresistant Staphylococcus aureus, Clostridium difficile, and vancomycin-resistant enterococci. Antimicrobial resistance among healthcare-associated pathogens is a world-wide problem and dominates all countries, yet teachings in the prudent use of antimicrobials has had little impact thus far despite major efforts to reduce antimicrobial resistance.

In summary, the IPC principles, but usually not the practice, are universally applicable. The IPC education programmes established by the Unit of IPC at Tygerberg Hospital in Cape Town are based on the initial needs assessment and take cognisance of the local teaching and working conditions. The build up of the IPC education programme has been gradual since 2005 and is expanding from healthcare facilities to the community, led by the dominant communicable diseases such as TB and HIV.

\section{IPC education programme}

\section{at Tygerberg Hospital, Cape Town}

Specific education structures in IPC have been established by the Unit of IPC at Tygerberg Academic Hospital to support the newly emerging IPC programme, established in 2005 and gradually expanded according to the needs of the hospital as well as the Western Cape Province. Qualification specific teaching includes other provinces from South Africa and students from the rest of Africa. The introduction of such programmes poses its own challenges which have to be addressed in accordance with local requirements.

\section{Challenges to setting up an IPC education programme}

When setting up a sustainable IPC education and training programme, there are challenges and our experience was no different. However, most of these have now been overcome and robust and sustainable IPC education programme is firmly on its way.

The IPC policies that did exist were out of date and were only included in the nursing procedure curriculum. The student nurses underwent intensive training at nursing colleges however the amount of time specifically allocated for IPC was limited. One of the challenges was to separate nursing procedures from IPC processes which required considerable support from senior management. At the time of introducing the IPC programme into our hospital it was acknowledged by senior management, mostly medical doctors, that they themselves had little IPC knowledge 
and also required training in this field.

This had to be taken into account when establishing a comprehensive IPC education programme as referred to later in this paper.

An acute shortage and rapid turn-over of staff resulted in a group of less knowledgeable workers, most of whom had not attended designated IPC courses where these existed. Informal instructions during ward rounds were not adhered to because staff changed so rapidly and many were not on the permanent pay roll - accountability was a challenge.

At the time of writing there were very few medically qualified doctors in South Africa and perhaps in the continent, trained in IPC. The reason was because IPC neither fell within a specific speciality nor was it a sub speciality in its own rights; IPC was considered primarily a nursing domain.

While named IPC nurse practitioners existed, not all of them were employed full-time or formally trained in IPC; IPC teams as such did not exist and there was little administrative support for those that did.
It was essential to convince governmental structures, such as provincial and national government, that the need for IPC structures and training was vital to the success of the IPC programme. Since this was viewed as an unnecessary expense because basic IPC was covered in the nursing training curriculum, champions had to found and nurtured.

English is not the first language for many of the IPC students and so the teaching programmes had to be geared towards being practical, visual with group discussions and class interaction. This was an exhausting but rewarding method of teaching and required the tutor to be knowledgeable and experienced in this method of teaching. The use of computer or web based learning was not possible because most of those attending the IPC training programmes did not have access to computers or the internet.

Lastly, only recently, since the WHO directives on establishing IPC structures, has IPC become important at national level. It is part of quality assurance and since it does not have a separate department, the progress is slow and disparate. In the last year, attention has turned to IPC as a major role player in the containment of TB and HIV and some guidelines have therefore been produced.

Fig 1: Structure of IPC training courses offered by the UIPC, TBH, Cape Town, S Africa

$*$ PDIC $=$ Postgraduate Diploma in IPC

\section{PDIC}

6-months

Fundamentals in

IPC for healthcare workers

In-house speciality

specific IPC training

5-day Introduction to IPC

for Healthcare workers

5-day Introduction to IPC

for Healthcare Managers 
Table I. UIPC Training 2005 - 2010

\begin{tabular}{|c|c|c|c|c|c|c|c|}
\hline Course & 2005 & 2006 & 2007 & 2008 & 2009 & 2010 & Total \\
\hline Postgraduate Diploma in Infection Control (PDIC) & - & 8 & 7 & 10 & 10 & 7 & 42 \\
\hline \multicolumn{8}{|l|}{ Decontamination and Sterilization Courses } \\
\hline Basic SSD & - & 20 & 41 & 63 & 34 & 30 & 188 \\
\hline Basic SSD (Namibia) & - & - & - & - & - & 35 & 35 \\
\hline Intermediate SSD & 5 & - & 9 & 16 & 13 & 12 & 55 \\
\hline Advanced SSD & - & - & 5 & 9 & - & 12 & 26 \\
\hline \multicolumn{8}{|l|}{ Short Courses } \\
\hline Introduction to IPC (TB \& HIV), Swaziland & - & - & - & - & 81 & - & 81 \\
\hline Train-the-Trainer in IPC (Swaziland) & - & - & - & - & - & 14 & 14 \\
\hline Basic TB-IPC Training for Managers (Swaziland) & - & - & - & - & 47 & - & 47 \\
\hline Basic IPC for Healthcare Workers (Namibia) & - & - & 29 & 25 & - & - & 54 \\
\hline IPC for Managers & - & - & - & - & - & 14 & 14 \\
\hline Tygerberg Hospital In-house Training & 198 & 269 & 306 & 707 & 235 & 369 & 2084 \\
\hline Ad hoc training & 53 & 31 & 20 & - & - & 210 & 314 \\
\hline
\end{tabular}

\section{Education programmes in IPC}

The IPC education programme was started in 2005. It is aimed at senior management and IPC practitioners, and structured in tiers in such a way that all healthcare workers are trained in at least the core elements of IPC such as standard precautions (Fig 1). The short courses and qualification courses have been registered with Stellenbosch University and are accredited by the Department of Education. Table I shows the number of students trained annually across the spectrum of IPC based on their educational needs.

\section{Postgraduate Diploma in Infection Prevention and Control (PDIC) ${ }^{9}$}

In 2005, the multidisciplinary Postgraduate Diploma in Infection Prevention and Control (PDIC) was established for those working in IPC for a period of two years or more. It is a two year part-time course structured as five modules. Each module is for ten weeks with two weeks of contact teaching and eight weeks of completing a log book and writing a project at ones place of work. The marks are divided equally for 1 . spot and written examination at the end of the two weeks, 2. logbook and 3. the project. An overall $50 \%$ is required to pass the module. The modules cover microbiology, risk management, decontamination and sterilization, hospital design and management and finally surveillance, audit and basic statistics. During the contact period, there are formal lectures in the morning and ward rounds, practical work and group discussions in the afternoon. This is a means of consolidating the information learnt and help to address pre-conceived notions and ingrained bad practice.

The students have to appoint a mentor at their place of work who should support and supervise their log book and project. The difficulty here is that most students cannot find qualified or knowledgeable mentors who understand IPC and many of them have to resort to 
consulting each other. A network of IPC practitioners is being established and is expected to mentor new students.

Entry level is a Bachelors Degree or equivalent and a minimum of two years in IPC, Microbiology or Epidemiology. Recognition for Prior Learning (RPL) is offered to registrars in Medical Microbiology for the first module. Each module may also be attended as stand-alone and a certificate of competence is issued if the student meets the examination requirements. The qualification is accredited by Stellenbosch University as are the short courses, and is equivalent to a Masters or NQF level 8 upon graduation.

Over the past five years, approximately 42 PDIC students (nurses, technologists and epidemiologists) have qualified, The UK based Hospital Infection Society gave $£ 10,000$ which provided ten bursaries to deprived provinces during the initial three years, thus boosting the training opportunity in these areas.

While the PDIC training has proven invaluable for the provinces of South Africa, some of the graduates have moved to higher administrative posts and are no longer practicing "hands-on" IPC. However many are acting as mentors for the current PDIC students; and the output is notably improved.

Apart from South Africa, the intake of students has extended to Zimbabwe, Namibia, Nigeria and more recently Cameroon. It is commendable that the foreign students are prepared to travel to Cape Town five times in the two years in search of IPC knowledge. The University of Zimbabwe is looking towards setting up its own IPC training programme in the future as is Namibia.

\section{Short Courses in IPC}

There are two basic five-day courses one for all healthcare workers and the other for executive managers which were started in 2007 and 2009 respectively.

\section{Introduction to IPC for Healthcare Workers}

This 5-day course is aimed at all healthcare workers from community clinics to inpatient facilities and encompasses basic microbiology, transmission, standard precautions, transmission-based precautions, hospital design and data collection. There is short examination at the end of the course and a certificate of competence is issued to those who pass with $50 \%$ marks and higher. The course is structured to integrate current practice into the training. Practical demonstrations and group discussions have a key role, therefore the emphasis on TB and HIV is notable and is used as examples for explaining good IPC processes. Thus far, there have been over 360 healthcare workers trained in Namibia, Swaziland and some parts of Kenya $(n=35)$ and Tanzania $(n=25)$. Training in Lesotho and Botswana is being considered. Reports from these countries are encouraging. In only three years, Namibia has gone from having only one IPC nurse in the main teaching hospital to an IPC practitioner in each large healthcare facility and one in a district hospital. The impact on IPC provision and practice is remarkable. While surveillance of $\mathrm{HAI}$ is lacking so far, the implementation of structures for IPC are probably the fastest in the southern African countries! Swaziland is now producing its IPC-TB guidelines and has got IPC structures in place towards improved programmes.

\section{Introduction to IPC for Healthcare Managers}

In 2009, all the executive managers of Tygerberg Academic Hospital attended a 5-day training course in IPC. The contents were tailor made for such a strata of healthcare workers and included the contents of the basic course plus cost effectiveness, emphasis on surveillance and importance of a robust and viable IPC structure. The ward rounds were an eye opener for most of them and had a major impact on accountability. Based on the reports of this course to the Provincial Government of the Western Cape administration, a second course was held for the provincial administrators including the Deputy Director General of Health! The impact was profound. IPC staffing structures were reinforced, a previously existing provincial IPC committee was boosted further and it was recommended that all executive managers in the Western Cape should attend this 5-day course. No examination was undertaken therefore certificates of attendance were issued.

\section{Fundamentals in IPC}

It became evident that several applicants had the enthusiasm but not the qualification or the background 
to attend the PDIC course. An intermediate course for those who wish to pursue IPC as a career or require preparation to enter the PDIC may enrol in the sixmonth Fundamentals in IPC course which will start in 2011. The modular structure will follow similar lines to the PDIC course but will be less intense with three two-week contact sessions and six weeks for completing the logbooks and projects. A certificate of competence will be issued for those that satisfy the examination requirements.

\section{Train-the-Trainer in IPC}

The Train-the-Trainer short course was developed in response to capacity building within health systems and to leave behind a robust and sustainable training capacity. The five day course requires that the trainers should have passed the Basic IPC course, or similar, with a good mark, have a demonstrable interest in training and be committed to building capacity among their peer group. The training consists of adult teaching methods, transferring knowledge by various means and learning to interpret and present information simply. The trainees are then asked to teach five students of their choice over the next two to three months, who do not necessarily have to be healthcare workers and could be members of the community or care givers. The tutors then examine the training material of, and students taught by, the trainers. If $50 \%$ of knowledge has been transferred accurately, the trainer is certified as competent to train. This method is useful in the cascading of essential knowledge within a very short time. Thus far, 14 trainers have enrolled in the course and are awaiting evaluation. This system applies well to the link nurse programme where essential information has to be passed on rapidly and accurately.

\section{Courses in Decontamination and Sterilization}

Decontamination and sterilization is a neglected area in Africa and lies under the jurisdiction of the operating theatres. It became evident that there was an urgent need to establish an independent career path in Sterile Services; in order to achieve this three tier modular course was established in 2007 as short courses.

\section{Basic Decontamination and Sterilization (D\&S Basic)} All operators in the Sterile Services Department (SSD) attend a half-day fundamental course. The D\&S basic course runs for five days with a further five weeks to complete a log book. The course has been tailor made for the SSD operators. Thus far over 188 operators have been trained.

The D\&S basic course has also been run in Namibia in 2010 and 35 staff have been trained there, and a further 40 will follow in 2011.

\section{Intermediate Course (D\&S Int)}

The intermediate course dove-tails into the PDIC module and the training is conducted together with the IPC students. Essentially it is for those SSD staff who have passed the D\&S Basic and show promise of moving towards supervisor or principal operator level. The 10-week course is intense and is taught by internationally recognised experts from the UK and The Netherlands. Thus far, over 80 SSD staff have been trained; the impact, according to their SSD managers, has been very positive and performance has much improved.

\section{Advanced Course (D\&S Adv)}

The D\&S Adv is structured towards those successful D\&S Int candidates who show potential of becoming SSD Managers; the number trained thus far are shown in Table I. The course is structured around risk management, administrative management and problem solving and is taught by international experts in sterile services and hospital engineering. The qualification is recognised by the Institute of Decontamination Sciences (UK). The outcome of this course has been that Tygerberg Academic Hospital has appointed the first qualified SSD Manager on the African continent in 2010! The impact on morale, process validation and improved patient care is noteworthy.

\section{Diploma in Hospital Technology (DHTec)}

There is a move towards establishing a Diploma in Hospital Technology (DHTec) which will include SSD as well as hospital technologists as teams to provide a quality sterile service with trained staff via an independent career path. The DHTec will be set up with the local Cape Town Peninsula University of Technology and will be divided into modules offered over a period of three years. 


\section{Structured In-house Training}

A local IPC manual has been developed and made available on the intranet which is accessible at each computer point in the clinical and non-clinical areas of the hospital.

As of 2011, systematic in-house training on each section of the IPC Manual will be conducted by a newly appointed training co-ordinator who has completed the PDIC. The one to two hour training will be provided for the entire team working in a particular speciality or sub-speciality but it is envisaged that it will be mainly attended by nurses. The outcome of this training will be measured by the annual audit currently carried out and by noting a reduction in the number of $\mathrm{HAl}$ in each speciality. In addition, IPC training will be provided to service and support workers, including porters, housekeepers, laundry workers, food handlers, and mortuary workers.

\section{Community based IPC programmes}

IPC is usually considered healthcare facility based but in LMI countries, the community plays an important role in reducing transmissible disease such as tuberculosis or blood borne viruses. Education programmes aimed at community workers, lay and peer supporters, care givers and community leaders, increases awareness and improves basic IPC practices. ${ }^{10}$ At the Unit for IPC, teaching programmes for the community have been developed and are being currently evaluated. The method of delivering this type of information differs from that which is given to professional healthcare workers although the underlying message remains the same. The philosophy behind community education programmes is that with knowledge come empowerment and taking responsibility for ones' own health and that of the family.

\section{Conclusion}

The impact of IPC training is visible throughout South Africa and other neighbouring countries. Apart from the courses run at the UIPC, Stellenbosch University, Wits University and KwaZulu Natal also run courses in IPC at the post graduate level. This combined effort of robust training should yield results in reducing $\mathrm{HAI}$, communicable disease transmission and improved community support.

Infection prevention and control should be promoted across all strata of healthcare workers but its success depends upon engaging the public in the process using simple concepts, taking into cognisance the local conditions and disease profiles to understand IPC.

\section{References}

1. Desai N, Philpott-Howard J, Wade J, Casewell M. Infection control training: evaluation of a computer-assisted learning package. Journal of Hospital Infection 2000; 44: 193-199.

2. Tavolacci MP, Ladner J, Bailly L, et al. Prevention of Nosocomial Infection and Standard Precautions: Knowledge and Source of Information Among Healthcare Students. Infect Control Hosp Epidemiol 2008; 29: 642-647.

3. Mehtar S. Lowbury Lecture 2007: infection prevention and control strategies for tuberculosis in developing countries lessons learnt from Africa. Journal of Hospital Infection 2008; 69: 321-327.

4. Sudhaker C, Rao S. Participatory hospital infection control training programme among nurses working in selected hospitals of Karnataka, India: a clinical practice improvement project. Journal of Hospital Infection 2008; 69: 292-294.

5. Zhang Z, Yamamoto T, Wu XN, Moji K, Cai GX, Kuroiwa C Educational intervention for preventing bloodborne infection among medical students in China Journal of Hospital Infection 2010; 75: 47-51.

6. Lee TC, Moore C, Raboud J M, Muller MP, Green K, et al. Impact of a Mandatory Infection Control Education Program on Nosocomial Acquisition of Methicillin-Resistant Staphylococcus aureus. Infect Control Hosp Epidemiol 2009; 30: $249-256$.

7. Rosenthal VD. Device-associated nosocomial infections in limited-resources countries: Findings of the International Nosocomial Infection Control Consortium (INICC), Am J Infect Control 2008; 36: 7-12.

8. Guerra CM, Ramos MP, Penna VZ, NR Goto JM, Santi LQ et al. How to educate health care professionals in developing countries? A Brazilian experience Am / Infect Control 2010; 38: 491-493.

9. Mehtar S. In Understanding Infection Prevention and Control. p 17. Ed S Mehtar 2010, pub JUTA, South Africa

10. Marjadi B, \& McLaws ML Rural Indonesian health care workers' constructs of infection prevention and control knowledge. Am J Infect Control 2010; 38: 399-403. 